\title{
PARAMETERS EFFECTING FORCED VORTEX FORMATION IN BLADE PASSAGEWAY OF DYNAMIC AIR CLASSIFIER
}

\author{
Martin AdamčíK ${ }^{a}$, Tomáš SvěrÁK ${ }^{a, b, *}$, Peter Peciar $^{c}$ \\ ${ }^{a}$ Brno University of Technology, Faculty of Mechanical Engineering, Heat Transfer and Fluid Flow Laboratory, \\ Technická 2896/2, 61600 Brno, Czech Republic \\ ${ }^{b}$ Brno University of Technology,Faculty of Chemistry, Institute of Materials Chemistry, Purkyñova 464/118, 612 \\ 00 Brno, Czech Republic \\ ${ }^{c}$ Slovak University of Technology in Bratislava, Faculty of Mechanical Engineering, Institute of Process \\ Engineering, Námestie Slobody 17, 812 31, Bratislava, Slovakia \\ * corresponding author: sverak@fch.vut.cz
}

\begin{abstract}
Air classification of particulate materials is a method of classifying particles into coarse and fine fractions based on their size, density or shape. The performance of the rotor air classifier is affected by operating parameters which include the classifier rotor speed, air inlet velocity and material feed rate. Effects of operating and structural parameters on turbulent flow field patterns inside of a dynamic air classifier are investigated. Increasing the computing power, together with new turbulence models and approaches to simulate complex fully turbulent problems by solving Navier-Stokes equations allows studying and capturing smaller flow structures and properties more accurately. Velocity vector maps for varying operating parameters are studied by means of numerical simulations. The experimental section includes a visualization of flow patterns and velocity vector maps in the rotor region by the use of the particle image velocimetry (PIV). Results are compared and discussed.
\end{abstract}

KEYWORDS: rotor air classifier, forced vortex, blade passageway, particle image velocimetry (PIV), numerical simulation CFD.

\section{INTRODUCTION}

Air classification of powder materials with the use of a dynamic air classifier is a method of separating particles into 2 or more fractions based on their size. These devices are used with a great success across a wide spectrum of industries ranging from chemical to pharmaceutical, due to their efficiency, sharp separation and the capability of adjusting product parameters. The dynamic state air classifiers development can be described through three generations, with centrifugal and cyclone classifiers as the first and second generation classifiers. The third generation is the rotor air classifier which can exert a radial forced centrifugal field [1, 2].

Active development of the material science continuously increases the requirements on even finer particle size distributions of powder materials. In the ideal classification, it would be possible to divide the mixture of sizes into two fractions, such that one lay wholly below a predetermined cut size and that the other lay wholly above it. Particles with the size equal to the predetermined cut size have a $50 \%$ probability of being collected together with either fine or coarse powders [3, 4]. However, such ideal process does not exist due to various stochastic factors such as particle collisions during which the coarse particles can capture the fine particles or fine particles can get into the aerodynamic wake formed behind other particles, or turbulent flow structures, and thus some finer ones get into the coarse product and vice versa [5-7]. One of the requirements to achieve the fine cut sizes and narrow particle distributions is a uniform flow field inside the air classifier. Several studies have been conducted on the structure of an air classifier, especially on the structure of a rotor cage [8,10]. Toneva et al. [11, 12] focused on experimental and numerical investigations of the single phase flow in the air classifier mill. They used the 2D-PIV to obtain the time-dependent 2Dvelocity distributions of the single phase flow and two phase flow in the regions of the impact element and the classifier. Feng et al. 1] obtained 2D vector graphs of the revolving flow field based on the tangential and radial velocity in the passageway of rotor blades. Liu et al. [13] measured the axial velocity of the gas flow in a gas cyclone by the 2D-PIV and 3D-PIV. Guizani et al. 14] successfully used the pitot tube to measure the air inlet velocity in the cross section of the pipe's air inlet. The detailed investigation and study of flow structures in the proximity of rotor blades and in the blade passageway provides valuable information and insight into the particle classification process. The presented paper deals with numerical (CFD) and experimental (PIV) investigations of fluid phase flow patterns and velocity vector maps inside the rotor air classifier. 


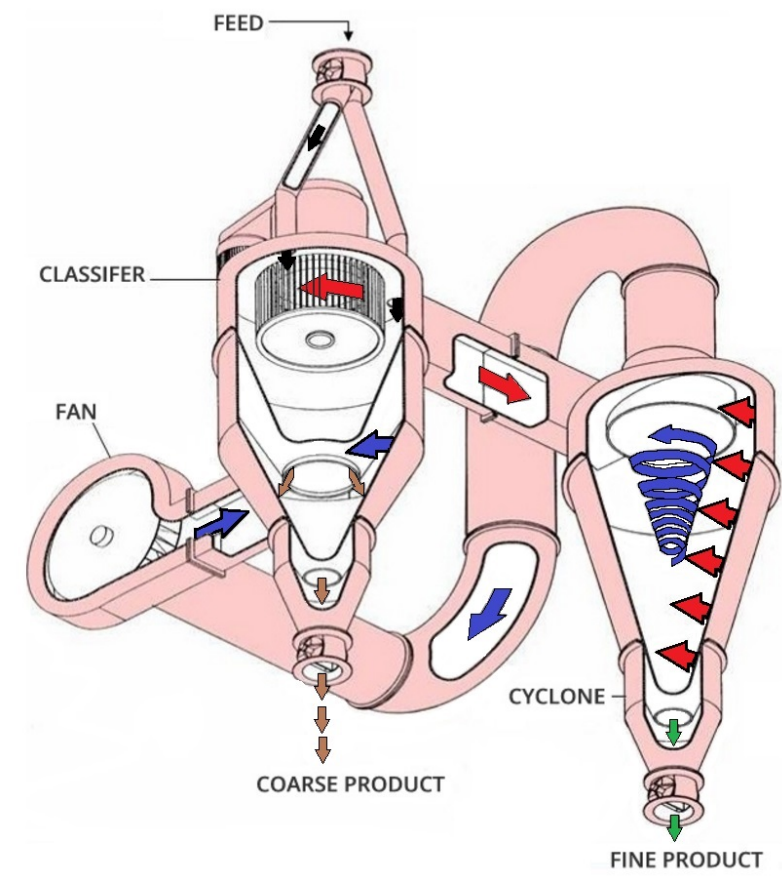

Figure 1. Indication of fluid and discrete phase flows. Black colour indicates feed material. Red colour indicates air flow saturated by fine particles. Brown colour indicates coarse particles. Blue colour indicates purified air. [15].

\section{EXPERIMENTS AND SIMULATION}

\subsection{EXPERIMENTAL SETUP}

Flow velocity investigations were performed on the MS5 wind sifter (Bradley Pulverizer Inc.). The wind sifter unit is installed in a closed circuit with a centrifugal fan re-circulating the air, which is used for the particle classification and transport. The upper chamber together with the inner cone form a cylindrical housing creating the centrifugal cross flow separation zone. The feed material enters through two feed ports in the top part of the upper chamber. It is then mixed with the air and different particles are accelerated to their individual terminal velocities through the airflow. Fine particles satisfying the classifier cut size parameter escape through rotor blade passageways and are carried away from the internal area of the rotor vertically up into the cyclone as shown in Figure 1

The classifier rotor is hanging vertically down and is equipped with 30 blades which are arranged in a straight radial pattern. The outline geometrical shape is a conical frustum with a larger diameter at the top. Height of the rotor is $250 \mathrm{~mm}$ and the diameter at the midplane is $275 \mathrm{~mm}$. The rotor is direct driven and the peripheral velocity is a variable with its maximum at $42 \mathrm{~m} / \mathrm{s}$.

Coarse particles are rejected and accelerated towards outer wall of the classifier. These particles are separated from the air stream through combined actions of the classifier rotor and the cyclonic separation

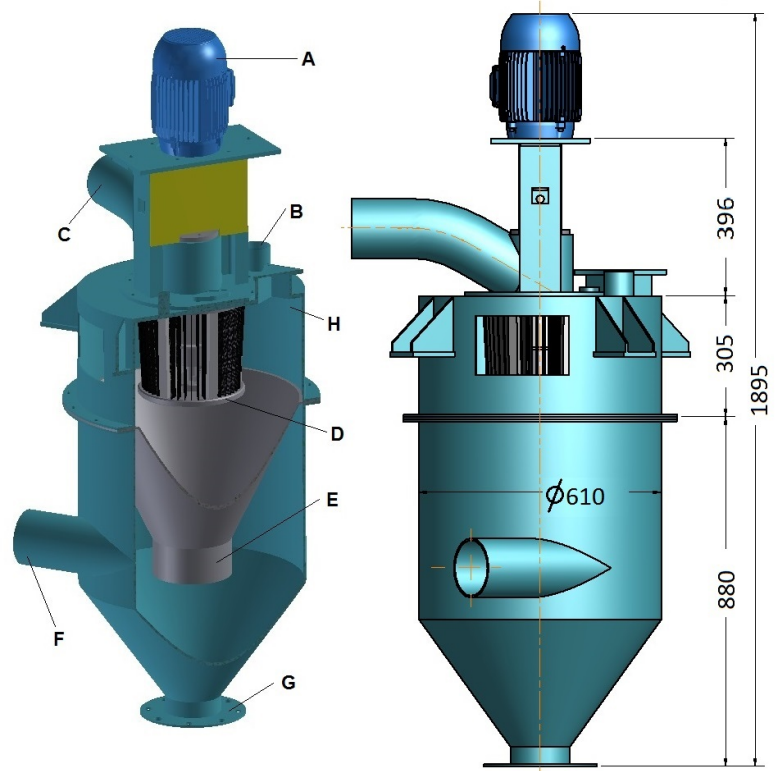

Figure 2. Outline dimensions in mm. Main parts: (A) motor; (B) feed inlet; (C) fine particles outlet;

(D) rotor cage; (E) inner cone; F-lower chamber;

(G) coarse particle outlet; $(\mathrm{H})$ upper chamber.

in the classifier body. The coarse fraction exits the lower chamber through the coarse particle outlet as shown in Figure 2 Clean air enters the lower chamber via a tangential inlet creating a swirling flow.

The air from the cyclone enters the centrifugal fan axially, and then it passes through the impeller where the kinetic energy of the impeller is used to displace the air radially towards the volute casing of the fan. It is then discharged at the right angle into the classifier tangential inlet. The fan is direct-driven by a 2-pole electric motor and the rotational speed of the impeller is controlled via a variable frequency drive. The fan used in the experiments is designed to deliver $1000 \mathrm{~m}^{3}$ /hour of air at a temperature of $20^{\circ} \mathrm{C}$, at $6575 \mathrm{~Pa}$ of a static pressure resistance with a $50 \mathrm{~Hz}$ electric motor power supply.

Modifications to the classifier upper chamber were carried out to allow the installation of the measuring equipment. These involved a creation of an opening in the top face of the classifier to allow a sCMOS camera gaining visual access to the blade passageway. An inspection opening on the side of the chamber was extended to allow the double laser head to illuminate the plane intersecting the region of interest. The inspection doors were modified to provide an air tight seal for the extended opening in the classifier and a flat frame was created for the laser sheet window. Both windows were sealed with a treated glass to provide an abrasion-resistant visual access and to create an air tight seal. Support frames for the laser assembly and camera were designed and fabricated as independent steel structures to isolate machine vibrations from the optical equipment. The laser frame allowed two angular and three linear adjustments and the camera 

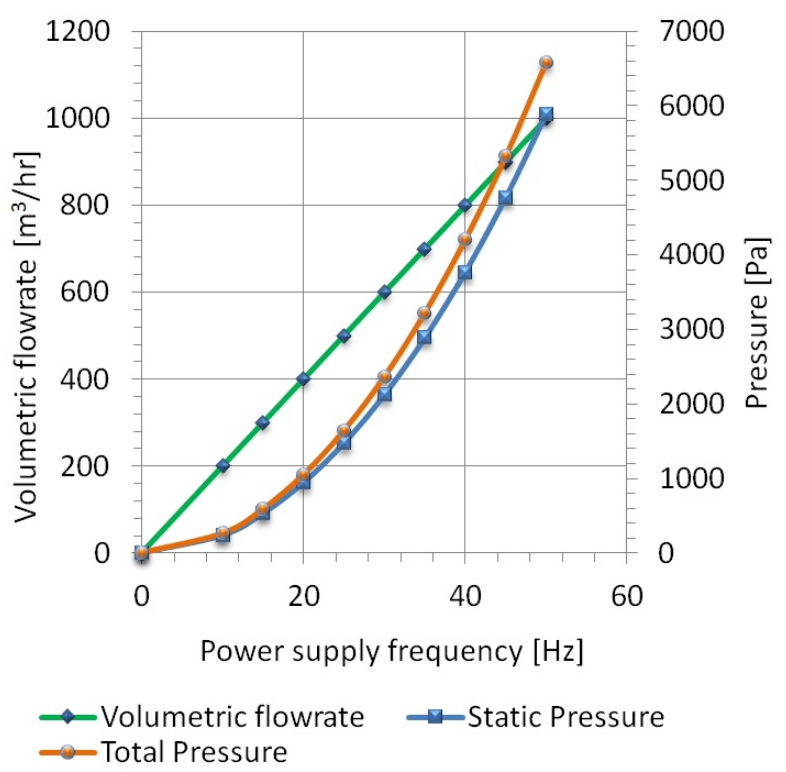

Figure 3. Flow rates, static and total pressure for $30 \mathrm{~Hz}$ and $40 \mathrm{~Hz}$ fan motor supply.

frame allowed adjustments in 6 degrees of freedom. The region of interest was located $300 \mathrm{~mm}$ from laser lens for the best laser sheet properties. Due to geometrical and spatial limitations imposed by construction of the classifier, the Scheimpflug lens was used to compensate for slight distortion of perspective.

\subsection{Air inlet Velocity measurement}

In order to increase the accuracy of the CFD simulation results, precise boundary conditions must be input into the numerical model. Fan affinity laws express relationships between the performance variables and were used to predict performance parameters of the fan. An incompressible version of these laws for maximum velocities in the region of $25 \mathrm{~m} / \mathrm{s}$ and low pressures were sufficiently accurate and the parameters were calculated [16]:

$$
\begin{aligned}
Q_{\mathrm{A}} & =Q_{\mathrm{B}}\left(\frac{n_{\mathrm{A}}}{n_{\mathrm{B}}}\right) ; \\
P_{\mathrm{AS}} & =P_{\mathrm{BS}}\left(\frac{n_{\mathrm{A}}}{n_{\mathrm{B}}}\right)^{2},
\end{aligned}
$$

where $Q$ is the volumetric flow rate, $n$ is the rotational speed and $P$ is the static pressure. Calculated values of the volumetric flow rate, static and total pressure developed by the fan, depending on the power supply frequency are presented in Figure 3 The fan delivers 600 and $800 \mathrm{~m}^{3} /$ hour at 30 respectively $40 \mathrm{~Hz}$ frequency of the power supply . For the inlet duct cross-sectional area, this represents inlet velocities of 10.83 and $14.43 \mathrm{~m} / \mathrm{s}$.

Effects of the classifier rotor rotation on the airflow can be compared to those of a centrifugal fan impeller. The classifier rotor displaces the air radially out due to the action of the centrifugal force, thus acting as an ineffective fan, against the main fan in the system. Increasing the tip speed of the rotor, therefore, increasing the system resistance curve (the pressure drop across the system). A manometer with a pitot tube was used to take static and total pressure readings in the inlet duct at different locations as seen in Figure 4

Readings were taken for 30 and $40 \mathrm{~Hz}$ frequency of the fan motor supply, each for 5 classifier rotor speeds. These were then converted into velocities with respect to

$$
v_{\mathrm{m}}=\sqrt{\frac{2\left(P_{\mathrm{T}}-P_{\mathrm{S}}\right)}{\rho}},
$$

where $\rho$ is density of the fluid, $P_{\mathrm{T}}$ is the total pressure, $P_{\mathrm{S}}$ is the static pressure and $v_{\mathrm{m}}$ is the velocity of the fluid in the duct, and averaged because velocities in the near wall region are lower than in the core region.

Figure 5 shows measured values of air velocities for 2 fan speeds and varying classifier rotor speed. The air inlet velocity increases with the decreasing classifier rotor speed. It can be observed that the calculated air velocities for 30 and $40 \mathrm{~Hz}$ fan motor settings at a maximum classifier rotor speed are lower than the measured values. This can be explained by the system resistance at the $50 \mathrm{~Hz}$ classifier setting that was lower than anticipated by the fan performance curve. Velocity readings were taken without the particle loading, which would have increased the system resistance and thus decrease the measured velocities.

\subsection{Blade PASSAGEWAY VELOCITY MEASUREMENT (PIV METHOD)}

The planar particle image velocimetry (2D PIV) is a non-invasive imaging technique consisting of several subsystems. It was used to measure velocities and to visualize flow patterns in the rotor blade passageway. The investigated plane was aligned with the expected flow direction and intersected rotor blades of the classifier.

The investigated flow was saturated with Di-EthylHexyl-Sebacate (DEHS) seeding particles. The particle concentration was adjusted by modifying the pressure and by closing or opening nozzles. Density of the DEHS is $0.91 \mathrm{~g} / \mathrm{cm}^{3}$ and its dynamic viscosity is 22-25 mPas. Aerosol generator was used to generate a polydisperse aerosol by atomizing the DEHS into particles in submicron range with an approximate lifetime of of 4 hours for a particle with size $0.5 \mu \mathrm{m}$. Particles travelling in the airflow were illuminated twice in the plane of the laser sheet.

Two-pulsed neodymium-doped yttrium aluminium garnet laser was used as a light source due to its high energy and short pulse capability. The $1064 \mathrm{~nm}$ laser beams were polarization-combined and then entered second harmonic generator producing a visible $532 \mathrm{~nm}$ light sheet with $200 \mathrm{~mJ}$ energy. The light beam was transformed into a $0.5 \mathrm{~mm}$ thin diverging sheet by an arrangement of spherical and cylindrical lenses. A double shutter Imager sCMOS camera (NoP 2560x1600) 

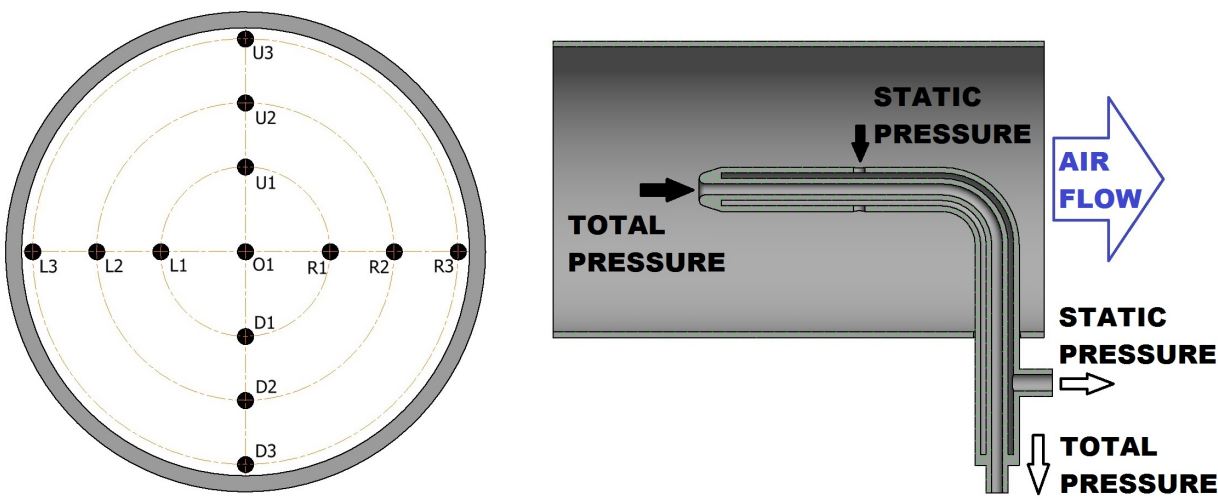

Figure 4. Schematic of pressure measurements with Pitot tube.

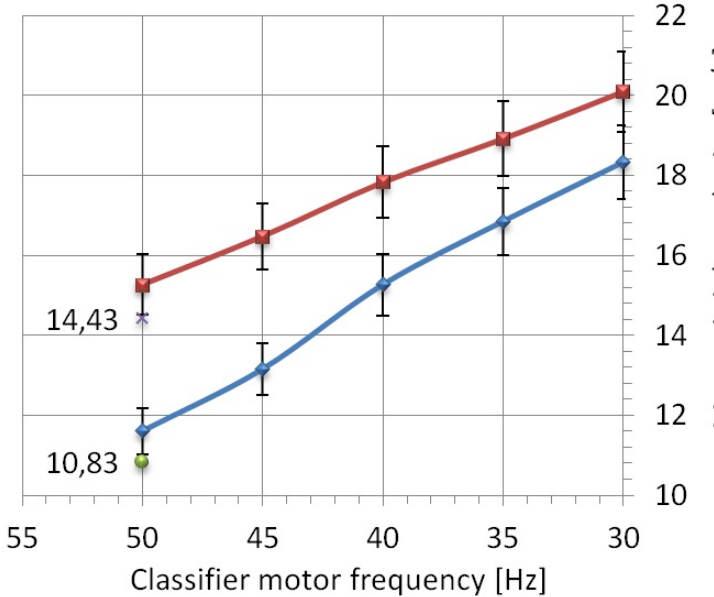

$\approx$ Fan mot. freq. $30 \mathrm{~Hz}$
$\rightarrow$ Fan mot. freq. $40 \mathrm{~Hz}$
$\rightarrow$ Calculated velocity $30 \mathrm{~Hz}$
$\approx$ Calculated velocity $40 \mathrm{~Hz}$

Figure 5. Measured and averaged air velocity in inlet duct.

was used to record the light shattered by particles on two consequent frames. The shutter speed was selected $3-5.5 \mu$ s so that the second frame captured the displacement of the original particles from the first frame. Each frame was divided into interrogation areas and average displacements of the group of particles that were mapped. The cross correlation was used to calculate the most probable displacement vector for a particular group of particles. Displacement vectors were converted into velocity vectors based on the known shutter time. Double frames were acquired at $15 \mathrm{~Hz}$ sampling frequency and 350-400 image pairs were recorded and averaged for each set of the operating parameters. Initial post-processing of images was carried out by the Davis software. Final processing of images was carried out by the Paraview software.

\subsection{CFD Simulation}

To carry out the CFD analysis of the investigated system a thorough decomposition of the problem is required. The classifier in the experimental part was

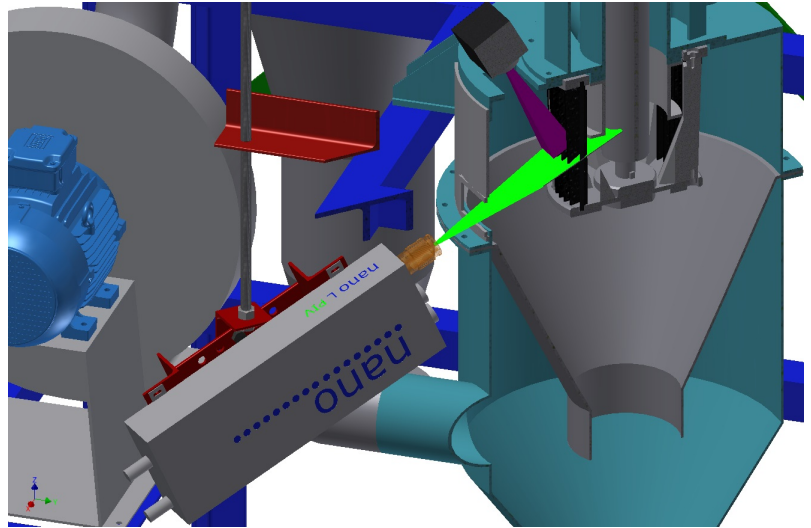

Figure 6. Assembly of classifier and PIV devices.
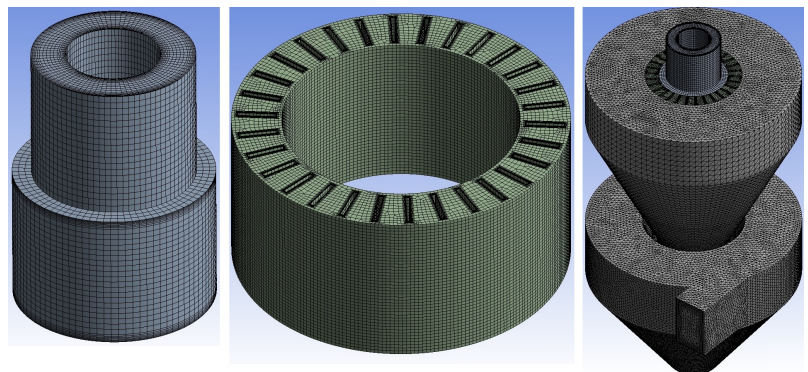

Figure 7. Mesh grids for outlet, rotor domain and internal body.

installed in a closed loop circuit. The loop was broken and the classifier itself was selected for the simulation. A 3-dimensional assembly model was created in Autodesk Inventor professional and geometry was simplified with respect to functional design of the device. The Finite Volume Method is a numerical technique that transforms the partial differential equations representing conservation laws over differential volumes into discrete algebraic equations over finite volumes [17.

Ansys meshing was used to discretize 3 solid bodies. A structured mesh with hexa elements was created for the outlet and rotor domain. The outlet domain mesh was created using multizone method. The mapped mesh type was built out of hexa elements whilst the free mesh type used tetra elements. Local body sizing with hard behaviour was applied and element size 


\begin{tabular}{|c|c|c|c|c|c|c|c|c|}
\hline & \multicolumn{2}{|c|}{ Number of } & \multicolumn{2}{|c|}{ Skewness } & \multicolumn{2}{|c|}{ Ort. Q. } & \multicolumn{2}{|c|}{ Aspect ratio } \\
\hline & nodes & elements & $\min$. & $\max$ & $\min$. & $\max$ & $\min$ & $\max$ \\
\hline Internal body & 352174 & 1069541 & 0.00018 & 0.84 & 0.21 & 0.99 & 1.16 & 14.01 \\
\hline Outlet & 68185 & 158516 & 0.0062 & 0.7 & 0.34 & 0.99 & 1.19 & 15.25 \\
\hline Rotor domain & 389180 & 353503 & 0.00045 & 0.72 & 0.47 & 0.99 & 1.01 & 16.54 \\
\hline
\end{tabular}

TABLE 1. Overview of quality indicators and elements in domain.

was selected to be $6.79 \mathrm{~mm}$. The inflation layer was created with respect to observed $Y^{+}$values. Rotor domain mesh was created by the multizone meshing. The mapped mesh type was built out of hexa elements whilst the free mesh type used hexa elements. The local body sizing with soft behaviour was applied and the element size was selected to be $3.2 \mathrm{~mm}$. An inflation layer was created around rotor blades with respect to observed $Y^{+}$values. The internal body was meshed with tetra and prism elements. Walls are the main source of the mean vorticity and turbuluence. Turbulence models are significantly affected by the no-slip condition valid for wall boundary conditions. Large gradients in the mean velocity cause a production of turbulent kinetic energy. The modelling of the flow in the near wall region significantly affected the numerical robustness of the solution. $Y^{+}$is a non-dimensional distance from wall,

$$
Y^{+}=\frac{y U_{\tau}}{\nu}
$$

where $Y^{+}$is the distance between the wall and first centroid, $U_{\tau}$ is the friction velocity and $\nu$ is the kinematic viscosity, and it describes how fine or coarse is the mesh in the wall bounded flows. A grid was created and optimized in iterative process to maintain $Y^{+}=30-60$ which are recommended values for a $k-\epsilon$ realizable turbulence model.

Table 1 shows mesh quality parameters and their values. A 3-dimensional flow of viscous fluid and the relation between velocity, pressure, temperature and density is described by Navier-Stokes equations. They are nonlinear partial differential equations representing mathematical statements of the conservation laws for mass, momentum in three directions. The conservation of mass is represented by the continuity equation which can be defined for compressible \& incompressible flows. The compressibility effects can be neglected for flows with a low Mach number

$$
M=\frac{U_{\max }}{c},
$$

where $U_{\max }$ is the maximum velocity in the fluid flow and $c$ is the speed of sound in the medium. The maximum velocity in the system was in the throat region and its value was $55 \mathrm{~m} / \mathrm{s}$. The speed of sound in the medium is $343 \mathrm{~m} / \mathrm{s}$ and for the given conditions the value of the Mach number is 0,16 . This value is significantly lower than the value of unity and therefore the continuity equation takes form for an incompressible flow.

Reynolds averaged Navier-Stokes (RANS) equations were solved by the commercial software Ansys Fluent together with additional 2 equations for turbulence.

The Reynolds number is used to predict the flow patterns and transitions from the laminar to turbulent flow regime. It represents a ratio of inertial to viscous forces in the fluid that is subjected to an internal movement due to different fluid velocities. Fluid friction generated by relative velocities propagates a developing turbulent flow. Increasing the viscosity of the fluid has just the opposite effect as more kinetic energy is absorbed and thus inhibiting the turbulence. The Reynolds number can be calculated for number of regions of the classifier, but the turbulence modelling is implemented for the whole domain. The term for the Reynolds number calculation takes a form designated for a cylindrical vessel agitated by a central rotating rotor:

$$
R e=\frac{\rho N D^{2}}{\mu},
$$

where $\rho$ is the air density, $N$ is the rotational speed, $D$ is the diameter of the rotor and $\mu$ is the dynamic viscosity of the air. The calculated Reynolds number was several orders of magnitude greater than considered threshold and the flow can be denoted as fully turbulent. Turbulent flows are time and coordinate dependent, diffusive and chaotic and causing rapid mixing. They involve 3-dimensional vorticity fluctuations with a wide range of time and length scales. KEpsilon realizable and RNG turbulence models, which were used to simulate effects of the turbulence, are two equation models which are the most commonly used models in industrial applications due to their reasonable accuracy, robustness and economy. The models solve the differential equation for the turbulent kinetic energy and dissipation rate and are valid for fully turbulent core flows where effects of the molecular viscosity are negligible. The K-Epsilon realizable model is the latest addition to this group of models and it differs from the standard K-E model in two aspects. It adopts a new formula for the turbulent viscosity calculation and a modified transport equation for the dissipation rate.

It is the only model from the group of K-E models that satisfies mathematical constraints for Reynolds stresses valid with the physics of a turbulent flow. The realizability of the model which essentially means the 


\begin{tabular}{lccccc}
\hline Fan & \multicolumn{5}{c}{ Inlet velocity $(\mathrm{m} / \mathrm{s})$ for classifier } \\
m. frequency \\
\hline & $30 \mathrm{~Hz}$ & $35 \mathrm{~Hz}$ & $40 \mathrm{~Hz}$ & $45 \mathrm{~Hz}$ & $50 \mathrm{~Hz}$ \\
\hline $30 \mathrm{~Hz}$ & 18.32 & 16.84 & 15.26 & 13.15 & 11.65 \\
$40 \mathrm{~Hz}$ & 20.10 & 18.92 & 17.83 & 16.48 & 15.27 \\
\hline
\end{tabular}

TABle 2. Velocities defined at velocity inlet boundary conditions.

positivity of normal stresses and Schwarz inequality for shear stresses is achieved by sensitizing the turbulent viscosity to the mean flow and turbulence. This model shows significant improvements over the standard K-E model for flows that involve rotation, vortices and a strong streamline curvature. The classifier had a weak rotating-stationary parts interaction and therefore the multiple reference frame approach was employed to simulate rotating blades. An advantage of this model is that a problem which is unsteady in an inertial frame, can be rendered as a steady problem with respect to the steadily rotating reference frame. It allows to model problems involving rotating parts by allowing moving reference frames in designated cell zones. The classifier flow domain was divided into 3 cell zones with interfaces defined in between them. Absolute velocity formulation was selected and no special transformation was required on the interface between fluid domains because velocities were stored in the absolute frame. In the absolute velocity formulation used by Ansys Fluent, the centripetal and Coriolis acceleration can be simplified into a single term and a generalized equation representing the conservation of momentum in a steadily rotating fluid domain can be written as

$$
\frac{\partial}{\partial t} \rho \vec{v}+\nabla\left(\rho \overrightarrow{v_{\mathrm{R}}} \vec{v}\right)+(\vec{\omega} \times \vec{v})=-\nabla P+\nabla \tau+\vec{F},
$$

where $\overrightarrow{v_{\mathrm{R}}}$ is the vector of the relative velocity (viewed in the moving reference frame), $\vec{v}$ is the vector of the absolute velocity (as viewed from the inertial frame), $\vec{\omega}$ is the vector of the angular velocity, $\tau$ represents shear tensor from viscous stresses. To compute a unique solution to a problem a set of physical boundary conditions was defined. These include information about known or approximated flow variables at the boundaries of the fluid domain. The inlet surface of the duct tangentially joining the lower chamber of the classifier was selected as the velocity inlet. The simulated velocity values for different fan and classifier rotor settings are shown in Table 2 The turbulence intensity at this boundary is low and thus the calculation is relatively insensitive to the inflow value. It was set to $4 \%$ which represents a reasonable value without a presence of experimental data.

Annulus surface at the top of the classifier was selected as the pressure outlet boundary of the fluid phase. The relative total pressure of $0 \mathrm{~Pa}$ was defined at the outlet boundary from the classifier. The cell

\begin{tabular}{cccc}
\hline $\begin{array}{c}\text { Supp. } \\
\text { freq. } \\
(\mathrm{Hz})\end{array}$ & $\begin{array}{c}\text { Classifier } \\
\text { rpm } \\
(1 / \mathrm{min})\end{array}$ & $\begin{array}{c}\text { Ang. } \\
\text { velocity } \\
(\mathrm{rad} / \mathrm{s})\end{array}$ & $\begin{array}{c}\text { Tip } \\
\text { velocity } \\
(\mathrm{m} / \mathrm{s})\end{array}$ \\
\hline $30 \mathrm{~Hz}$ & 1785 & 186.92 & 25.23 \\
$35 \mathrm{~Hz}$ & 2081 & 217.92 & 29.42 \\
$40 \mathrm{~Hz}$ & 2380 & 249.23 & 33.65 \\
$45 \mathrm{~Hz}$ & 2685 & 281.17 & 37.96 \\
$50 \mathrm{~Hz}$ & 2975 & 311.54 & 42.06 \\
\hline
\end{tabular}

TABLE 3. Simulated rotor speeds.

zone located between the internal body and the outlet zone was a rotor blade zone. Two interface surfaces were defined at this cylindrical zone where the first one was coupled with internal body and the other was coupled with the outlet cell zone. In order to compute fluxes between different cell zones, an interface definition was required. The rotor cell zone was defined with rotating reference frame. 5 classifier rotor speeds were simulated for different air inlet velocities as seen in Table 3

\section{EFFECT OF OPERATING AND STRUCTURAL PARAMETERS}

\subsection{Forced VORTEX FORMATION IN BLADE PASSAGEWAY}

Velocities and flow structures in the blade passageway were measured via PIV for 10 cases with varying operating parameters as per Table 2 and Table 3 Measured velocities and visualized flow structures were used to verify the accuracy of the numerical model. Before every data acquisition, the particle displacement vector was evaluated manually. The particle shift for further processing was selected in a region of 4-8 pixels. For each measurement campaign, 350-400 image pairs were initially processed and averaged with the Davis software as seen in Figure 9 A sliding background subtraction filter with filter length of 8 pixels was used to minimize the intensity fluctuations in the background due to reflections. Polygonal geometric mask was created at a fixed location of the laser light sheet. A multi-grid interrogation with a final window size of $48 \mathrm{x} 48$ pixels was defined with a $75 \%$ overlap and a final vector grid spacing of 12 pixels. Each cross-correlation of a single pair of interrogation windows resulted into a single displacement vector. All vectors were then combined to form a final $2 \mathrm{D}$ vector field of the image. A median filter with an interpolation and smoothing were used to fill up empty spaces after the outlier removal. Velocity vectors were recorded in an inertial reference frame. Velocity vector based on the angular velocity was subtracted to transform vectors from the inertial to non-inertial reference frame of the rotor. Averaged images and data files were then postprocessed by the Paraview software, where several layers of extraction filters, calculator filters, threshold 

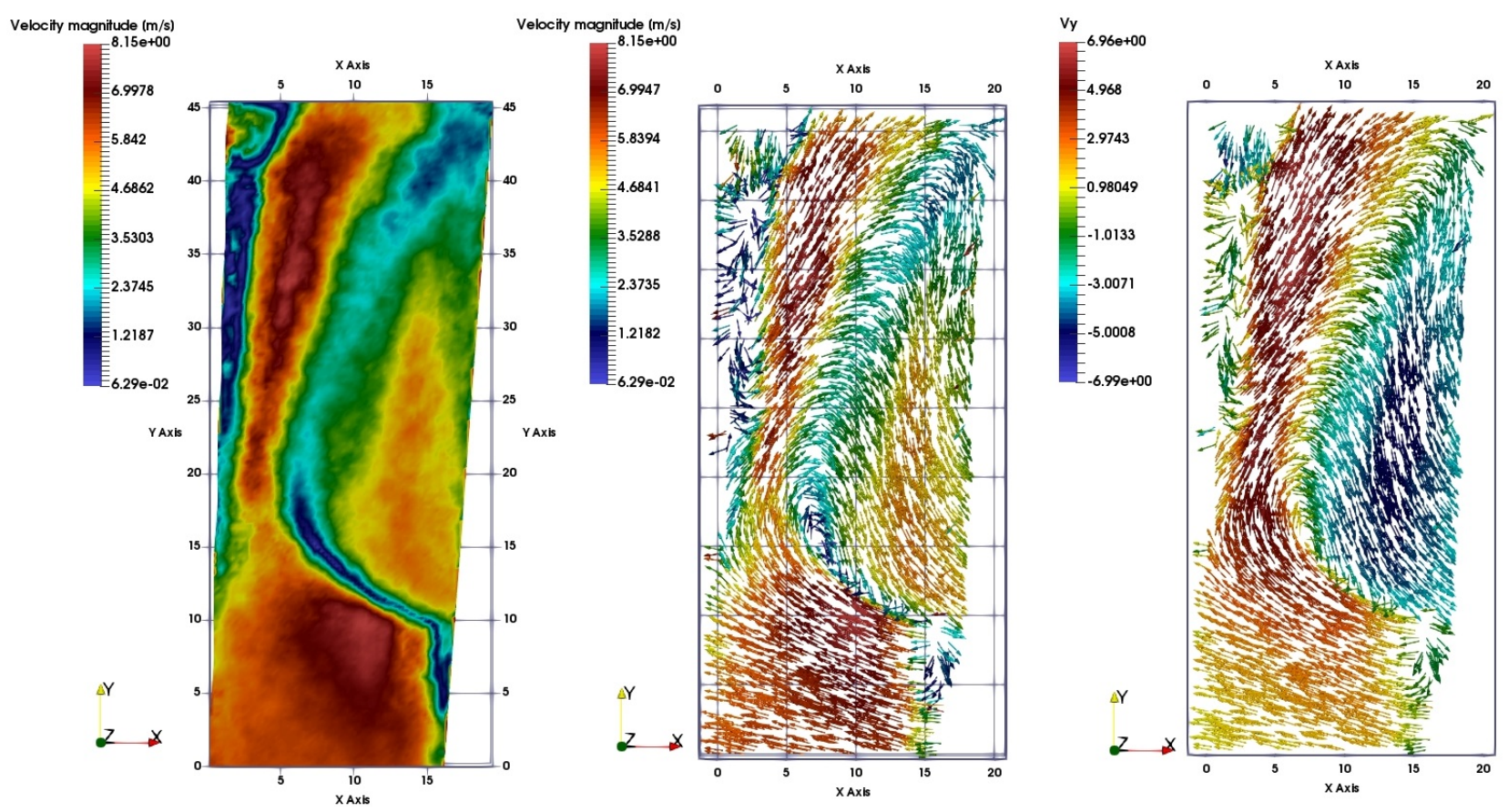

FiguRE 8. Flow field in blade passageway by maximum velocity; vector field by maximum velocity; vector field by maximum radial velocity (for 30-30 settings).

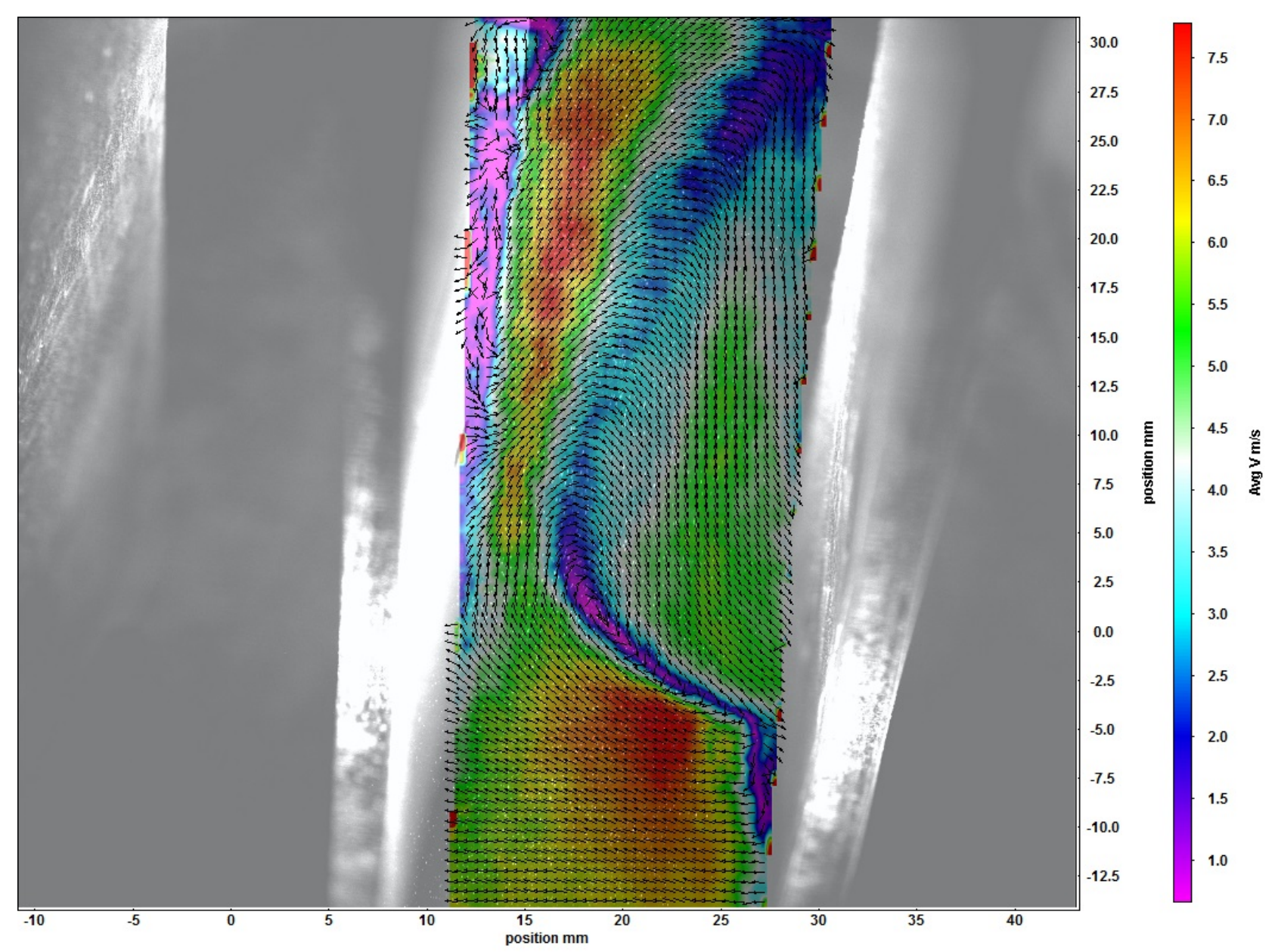

Figure 9. Averaged velocity vector field output from Davis software. 


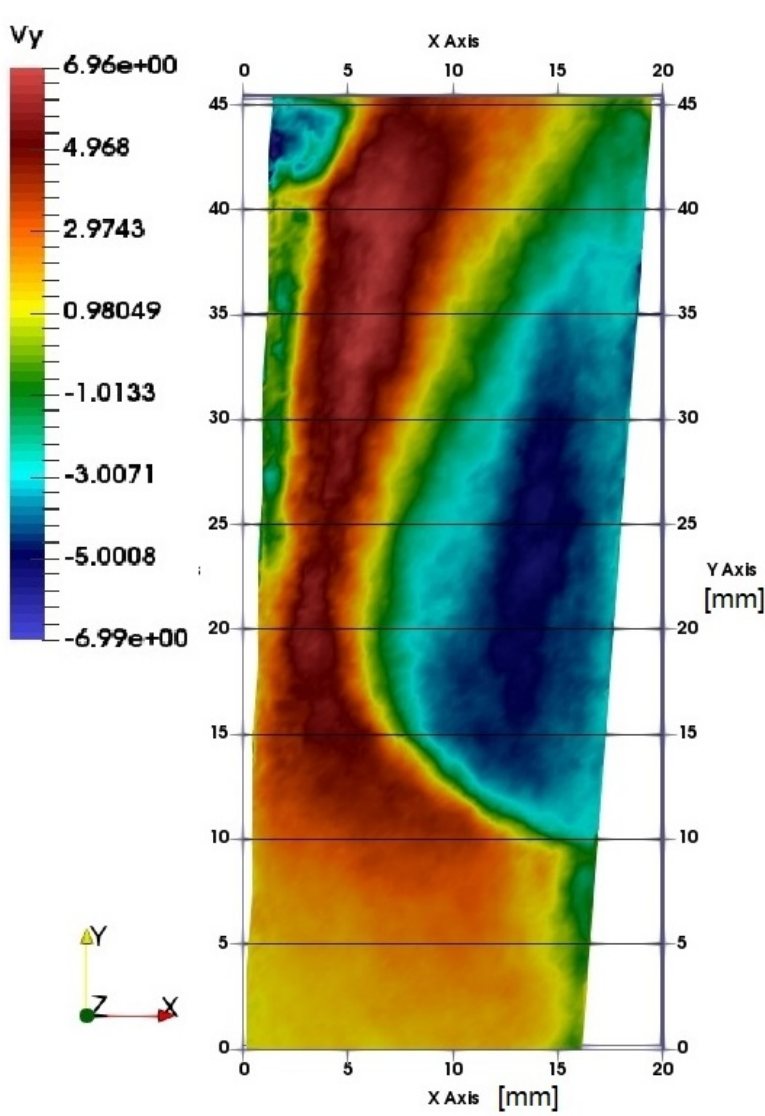

FiguRE 10. Radial contours for 30-30 setting.

filters, glyph filters Delaunay 2D filters were applied. The blade passageway was mapped by 9 sampling lines with a spacing of $5 \mathrm{~mm}$ in the radial direction. The classifier rotor cage spin anti-clockwise and therefore the right edge of the image represents the leading blade whilst the left edge represents the trailing blade. There were 30 blades evenly spaced along the circumference of the rotor, they were $30 \mathrm{~mm}$ deep with a $20 \mathrm{~mm}$ wide passageway. The outside pressure edge of the blades starts at $Y=10 \mathrm{~mm}$ and ends at $Y=35 \mathrm{~mm}$.

The air entered the blade passageway from the area outside the blades, $(Y=0-10 \mathrm{~mm})$. Here the flow direction was almost tangential with a very weak radial velocity component. This is beneficial for the particle classification and it has been proven by various researches and experiments. Maximum velocity was observed in this region and its value for 30-30 settings was $8.15 \mathrm{~m} / \mathrm{s}$. The forced vortex formation was confirmed in the area behind the leading blade. It was the area where the airflow had an opposite, outwards direction. This phenomenon resulted into a restricted passageway and as a result, the airflow accelerated in the unrestricted region passing alongside the forced vortex.

As the air entered the blade passageway the tangential velocity component became smaller and the radial became a dominant component. The sign convention is that the radial velocity is positive in the unrestricted

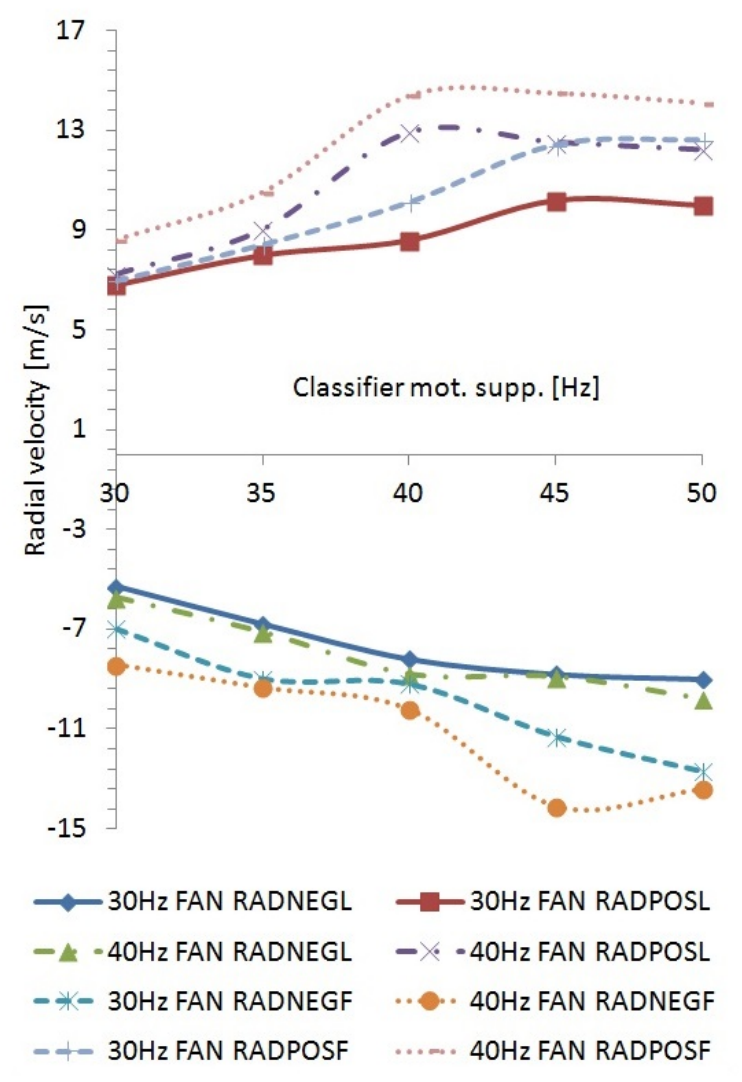

Figure 11. Maximum and minimum radial velocities for varying rotor speed obtained from sampling lines and from data filtration.

region in the inwards direction and negative in the outwards direction. Radial velocity contours are shown in Figure 10 and the maximum radial velocities were observed in the second half of the depth of the passageway.

The location, where the radial velocity changed from positive to negative, was established by intersecting profiles with zero value. Velocity profiles were slightly distorted in the proximity of the blades due to the laser light reflections and due to imperfect geometric constraints between the classifier rotor, laser and the camera. This resulted into a geometric mask which was not completely rectangular. The dat. file was then further filtered to establish maximum and minimum radial velocities as those lay outside the sampling lines. Radial velocities collected from sampling lines and those obtained by the filtration are presented in Figure 11. Area of the negative radial velocity in the blade passageway was bordered by locations where it changed from positive to negative. These positions are plotted for various operating parameters with respect to passageway dimensions as shown in Figure 12 For the fan setting at $30 \mathrm{~Hz}$, the negative radial velocity (eddy recirculation area) was decreasing in size with the increasing classifier rotor speed. The most significant size change occurred between 30 and $35 \mathrm{~Hz}$. This trend was less profound for the fan setting at $40 \mathrm{~Hz}$. 


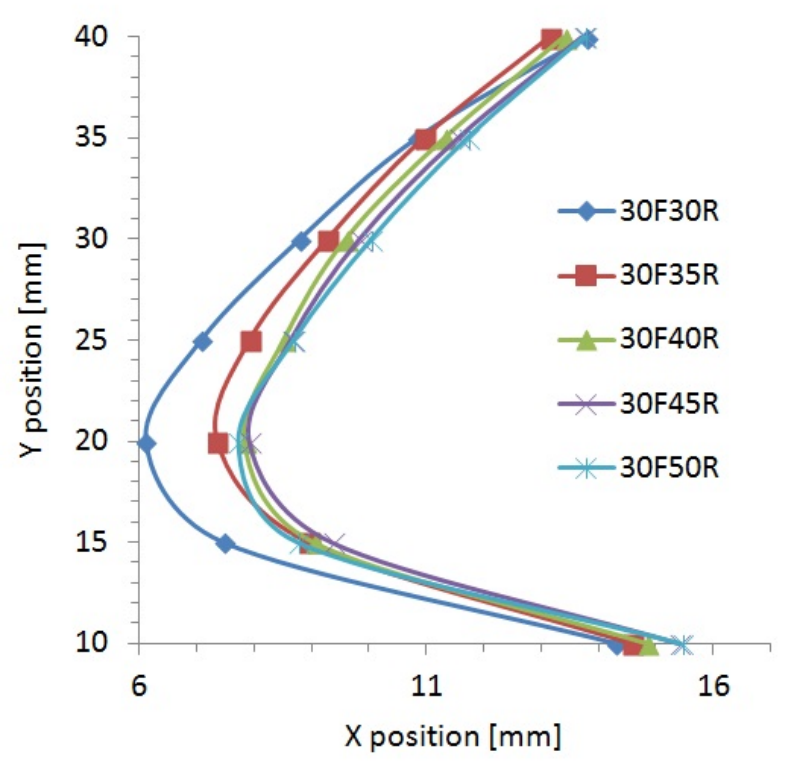

FiguRE 12. Position of inversion points for different operating parameters.

Two sets of numerical simulations with 30 and $40 \mathrm{~Hz}$ fan setting with five simulations in each set for the varying rotor speed were carried out. The numerical simulation predicts a formation of vortex in the area behind the leading blade for all the ten cases with the anti-clockwise rotor rotation. As the airflow exits the passageway, its radial component became minor whilst the tangential component increased.

In several cases it was observed that streamlines, which exited the blade passageway then repeatedly reentered the following passageway. This is a negative factor as the streamlines represent lines with tangents always parallel to the local velocity and therefore it meant that some of the air was re-circulating in an undesired pattern. In all cases, the radial velocity in the area right behind the leading blade was negative with an outwards direction. This is a negative factor as the airflow magnitude and direction is one of the two major variables determining the fate of the particles.

Figure 14 shows values of radial velocities obtained from the numerical simulation and their comparison to values obtained by the PIV measurement. $\mathrm{Nu}-$ merical simulation correctly predicted an increasing magnitude of both positive and negative radial velocities with an increasing rotor speed. The actual magnitudes varied due to several reasons. It was found out that radial velocity profiles in different blade passageways vary with geometrical position of the passageway with relation to other geometrical realities and design of the classifier. It has been observed that from total number of 30 passageways, 15 of them were described by a more profound vector field with higher velocities whilst the other half of the rotor had radial velocities significantly lower, as seen in Figure 15. It was found that the axis of the rotation of the swirling flow was not concentric with

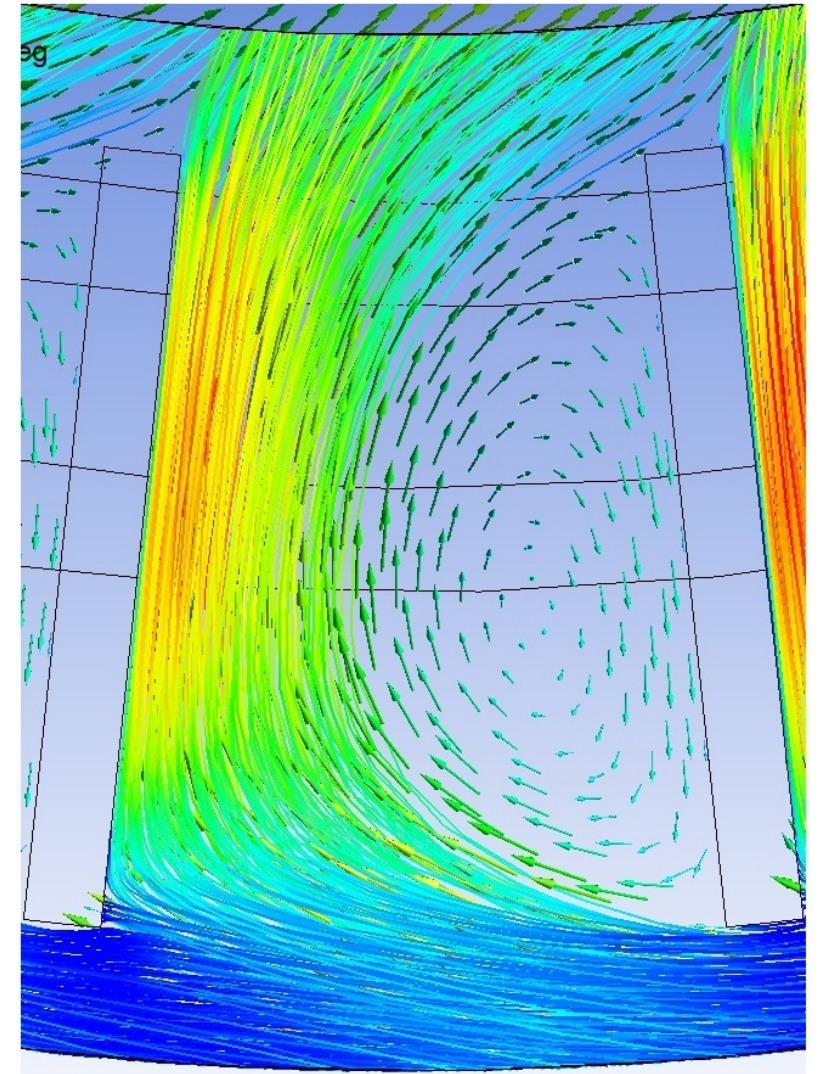

FiguRE 13. Vector map in examined plane overlaid by streamlines for $30 \mathrm{f}-30 \mathrm{r}$ setting.

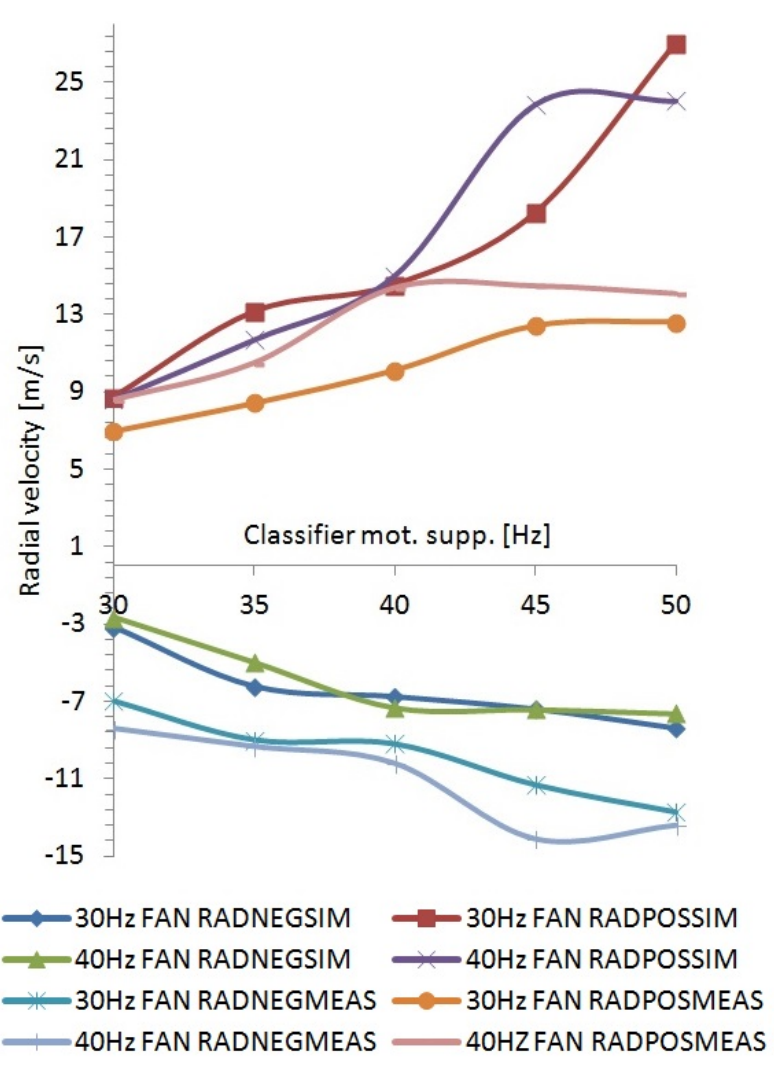

FIgURE 14. Simulated positive and negative radial velocity and comparison with measured data. 

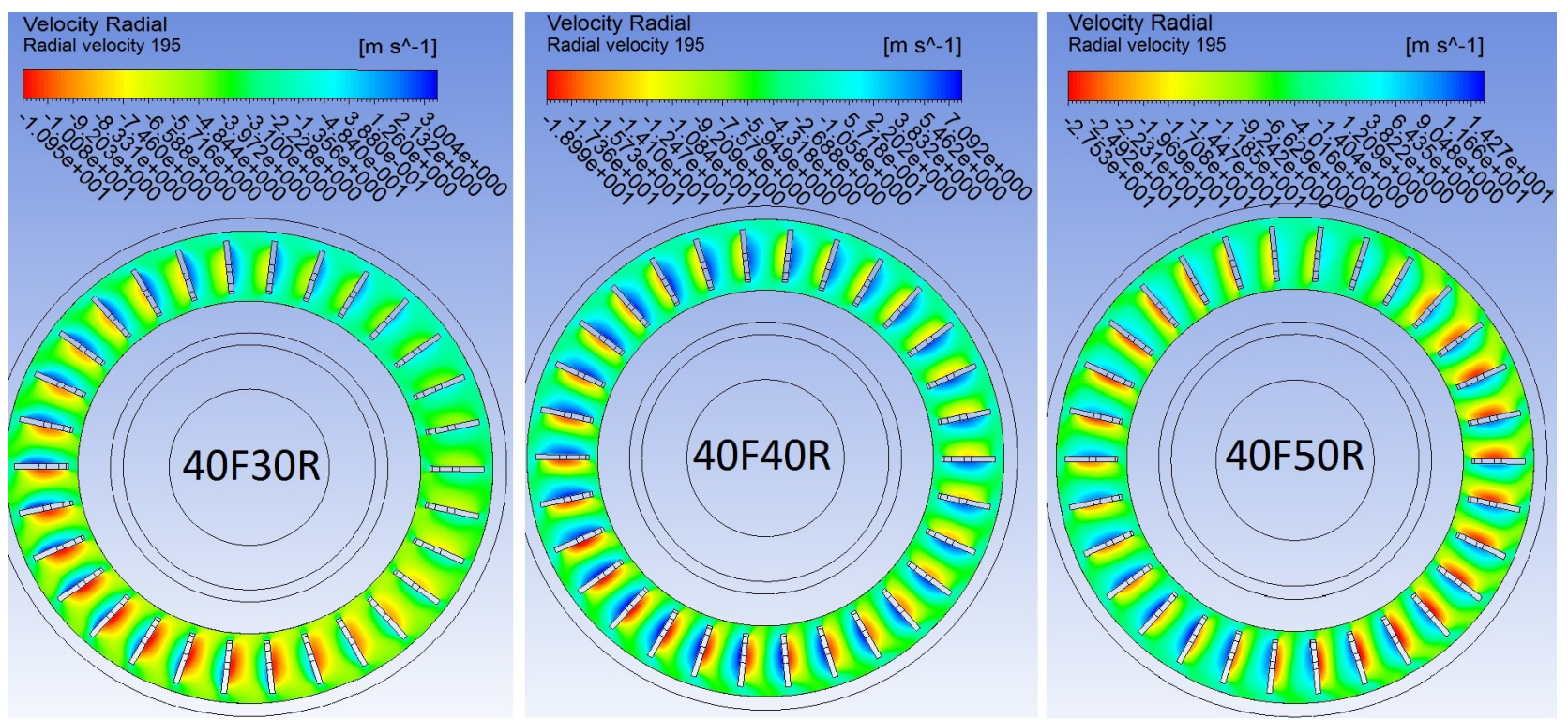

FIGURE 15. Section through rotor on radial velocity for varying operating parameters.

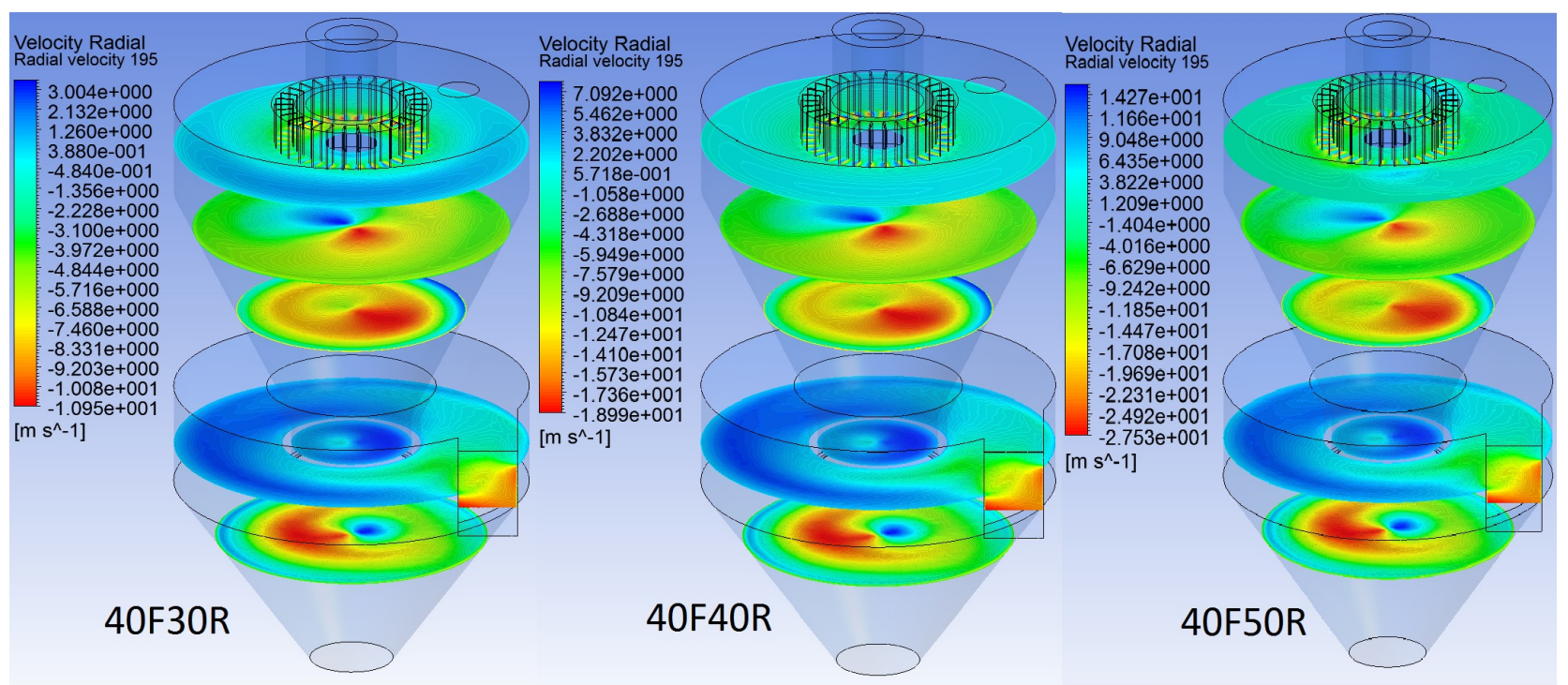

FiguRE 16. Radial velocity contours for selected settings on horizontal planes at different levels.

the axis of the cylindrical body. Position of the axis of the rotation was affected by the position of the lip leakage. Bottom section of the classifier had a tangential air inlet connected to the outlet of the centrifugal fan.

The tangential inlet created a swirling flow pattern in the bottom section of the classifier, which was separated from the top section by the inner cone. It was observed that the throat of the inner cone leaked the air into the top section in a specific location which determined the position of the axis of the rotation of the swirling flow and consequently determined which half of the classifier top body contained mainly positive and which mainly negative radial velocity components. These patterns were distinctive mainly in the area under the rotor as seen in Figure 16

It was observed that at some locations, the radial velocity was negative, and therefore pointing towards walls of the classifier body. This is an undesired flow pattern due to negative effects on the classification efficiency.

Figure 17 shows the position of the lip leakage in the area $1 \mathrm{~mm}$ under the lip of the inner cone throat. It was found out that in the lower section of the classifier, the leakage position was determined by the position of the tangential air inlet with respect to the cylindrical body of the classifier.

Uniform and organized flow field has positive impact on the classification efficiency and sharpness of the cut. The classification of particles with sizes below 5 microns requires high rotational speeds. Implication of a high rotational speed that must be considered is a creation of a forced vortex with an increased intensity. A rapid development of the computing power of processors allows new techniques to be used for the flow field analysis. Number of researchers carried out 

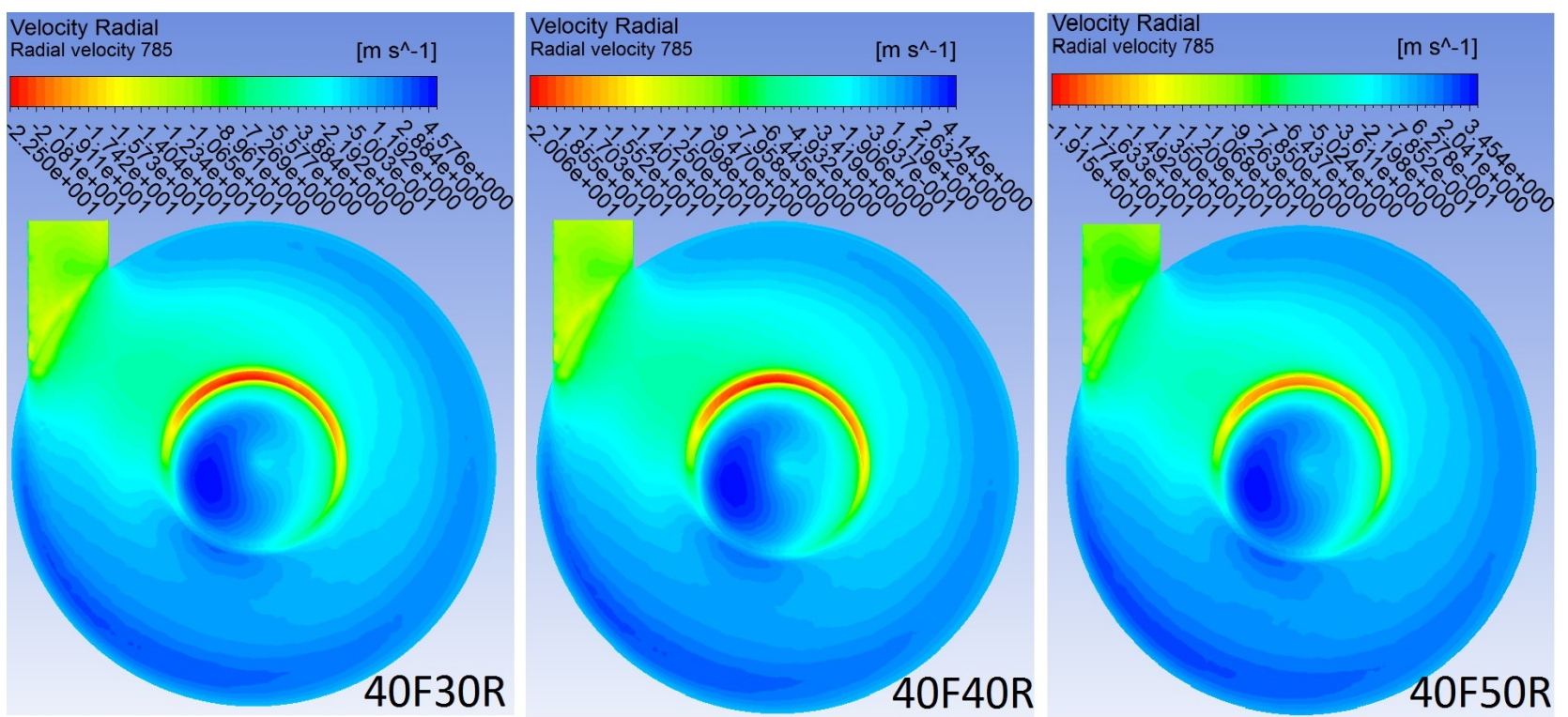

FiguRE 17. Lip leakage for $40 \mathrm{~Hz}$ fan speed and $30,40,50 \mathrm{~Hz}$ rotor speed.

simulations using the computational fluid dynamics approach with varying levels of accuracy. This can be partially attributed to the selection of turbulence models and current stage of their advancement. The $\mathrm{K}$-epsilon realizable model is the latest addition to the family of 2 equation turbulence modelling and it has improved the accuracy for simulations involving swirling flows.

Feng et al. [1] displayed a $2 \mathrm{D}$ vector graph of the rotating flow field in his study. He found out that vortex is not convenient for the classification as the fine particles can get into coarse ones and vice versa. He calculated that the intensity of the vortex is smallest when the tangential velocity of the exterior of the rotor blade and tangential velocity of the air flow in the rotor exterior edge are approximately equal. Guizani et al. [14] also focused on the vortex in the classifier. He identified that it is not the vortex which has a negative impact on the classification performance, but it is its breakdown. He further described two types of a vortex breakdown, namely the bubble and spiral breakdowns. In the air classifiers, the occurrence of a bubble type breakdown is dominant and it is the main factor responsible for the creation of recirculation areas. These recirculation flows are further responsible for the entrainment of fine particles in the coarse fraction. This phenomenon is called the fishhook effect. Galk et al. [8] studied the importance of the forced vortex and its influence on the fine particle separation. The research displays the size and position of the vortex in the passageway between blades depending on the rotational speed and radial velocity. These findings are in an agreement with a technical practise. There is, however, only a small number of researches which describe the flow field in the rotor region by the use of direct vector measurements. This may be attributed to the difficulties related to the PIV implementation into rotor air classifier. To name a few, environment in rotor air classifier needs to be strictly controlled for PIV and thus eliminating the possibility of applying PIV to industrial installations. The aspects of smaller laboratory versions of rotor air classifiers make it very difficult to implement the PIV equipment due to their dimensional size. Also the cost of the equipment needed to realize PIV measurements is significant and thus preventing its wider use. However, the PIV measurement and its results give us however an invaluable insight into flow physics and form a great data set for a validation of a numerical model.

\section{Conclusion}

Formation of a forced vortex in the blade passageway of a rotor air classifier was investigated under varying operating parameters. Numerical simulations were carried out and velocity vector maps describing the vortex were measured and visualized by a non-invasive method - PIV. Positive and negative radial velocities of the vortex increased with increasing the rotor speed and increasing the air inlet velocity. The particle classification is affected negatively by the greater intensity of the re-circulation area in the blade passageway. It was observed that the vortex intensity was not uniform in all 30 passageways. Position of the inner cone lip leakage depends on the upstream geometry where, as a consequence, the axis of the rotation of the swirling flow was not coincident with the axis of the rotation of classifier rotor. This resulted into mainly positive radial velocity components in one half of the classifier and mainly negative ones in the other half.

\section{ACKNOWLEDGEMENTS}

The authors appreciate the assistance of Bradley Pulverizer Co. in providing the testing classifier unit and in providing the premises and financial support for the PIV equipment hire. 


\section{REFERENCES}

[1] Y. Feng, J. Liu, S. Liu. Effects of operating parameters on flow field in a rotor air classifier. Minerals Engineering 21:598-604, 2008.

[2] L. Guo, J. Liu, S. Liu, J. Wang. Velocity measurements and flow field characteristic analyses in a rotor air classifier. Powder Technology 178:10-16, 2007.

[3] P. 83. Particle technology. The Institute of Chemical Engineers symposium series. ISSN 0307 - 0492, 69.

[4] Y. Yu, J. Liu, K. Zhang. Establishment of a prediction model for the cut size of rotor air classifier. Powder Technology 254:274-280, 2014.

[5] R. G. Holdich. Fundamentals of Particle Technology. Midland Information Technology and Publishing, 2002.

[6] L. Karunakumari, C. Eswaraiah, S. Jayanti, S. S. Narayanan. Experimental and numerical study of a rotating wheel air classifier. American Institute of Chemical Engineers Journal 51 (3):776-790, 2005.

[7] M. Shapiro, V. Galperin. Air classification of solid particles: a review. Chemical Engineering and Processing 44:279-285, 2005.

[8] J. Galk, W. Peukert, J. Krahnen. Industrial classification in a new impeller wheel classifier. Powder Technology 105:186-189, 1999.

[9] Q. Huang, J. Liu, Y. Yu. Turbo air classifier guide vane improvement and inner flow field numerical simulation. Powder Technology 226:10-15, 2012.
[10] R. Liu, J. Liu, Y. Yu. Effects of axial inclined guide vanes on a rotor air classifier. Powder Technology 280:1-9, 2015.

[11] P. Toneva, K. Wirth, W. Peukert. Grinding in an air classifier mill - part ii: Characterisation of the two-phase flow. Powder Technology 211:28-37, 2011.

[12] P. Toneva, P. Epple, M. Breuer, et al. Grinding in an air classifier mill - part i: Characterisation of the one-phase flow. Powder Technology 211:19-27, 2011.

[13] Z. Liu, Y. Zheng, L. Jia, et al. Stereoscopic piv studies on the swirling flow structure in a gas cyclone. Chemical Engineering Science 61:4252-4261, 2006.

[14] R. Guizani, I. Mokni, H. Mhiri, P. Bournot. Cfd modelling and analysis of the fish-hook effect on the rotor separator's efficiency. Powder Technology 264:149-157, 2014.

[15] M. Adamčík. Mechatronics of classifying of micro particles by air separation. University of Technology, Brno, 2010.

[16] E. b. R. J. Buffalo Forge Company. An Engineer's Handbook On Fans and Their Applications, Eighth Edition. Fan Engineering, Buffalo New York, 1983.

[17] F. Moukalled, L. Mangani, M. Darwish. The Finite Volume Method in Computational Fluid Dynamics, vol. 113. Springer International Publishing, 2016. 